\title{
The calculation of the depth of penetration for projectiles into rock
}

\author{
M. Y. Wang, Z. Shanbiao \& Z. Daliang \\ Engineering Institute, University of Science and Technology, China
}

\begin{abstract}
In this paper, an analytical model is used for characterizing the deformation and failure behavior of the rock in the process of penetration. The scaling law in penetration and perforation is uncovered, resulting in a formula for the computation of penetration on wide scales on a sound physical background.

Key words: penetration, scaling relation, critical stress-intensity factor.
\end{abstract}

\section{Introduction}

The problems of penetration and perforation into rock by projectiles have been studied for a long time, but to date, there has been not yet a completely satisfactory method for solving such problems due to their complex characteristics such as elastic and non-elastic deformations concomitantly occurring accompanied by crack propagation in the process of penetration and perforation. As a result, empirical formulas derived from experimental data consist in the main part of research products [1-3]. However, such purely experimental approaches have some defects, the most significant of which is that the results extrapolated from these formulas are not reliable where the change is extremely large in values of radius of projectiles or in values of other original parameters beyond the range of experiment. Hence, the major empirical formulas have a limited range of application and an alternative procedure should be developed to cover a wider scale.

In addition to empirical procedures, there are several theoretical models which can be classified into four groups as follows [4-6]: The first one is the rigid-plastic model, the second one is the classic spherical cavity expansion model and the third one is the numerical model. 
In this paper, the scaling law in penetration and perforation is uncovered, resulting in a formula for computation of penetration on wide scales on a sound physical background.

\section{Penetrating resistances}

The average strength of the projectiles is significantly larger than from that of the rock media. Thus, for simplicity in this paper, the projectiles is approximated by an absolute rigid body and assumed to have a conical-shaped end and penetrate vertically into the rock media.

The equation of motion for materials in the pulverized zone is described in a spherical polar coordinate as

$$
\rho_{0}\left(\frac{\partial v}{\partial t}+v \frac{\partial v}{\partial r}\right)=\frac{\partial \sigma_{r}}{\partial r}+\frac{2\left(\sigma_{r}-\sigma_{\theta}\right)}{r}
$$

where $\rho_{0}$ is the initial density of the material, $v$ is the radial velocity of the particle, and $\sigma_{r}, \sigma_{\theta}$ are radial and lateral components of stress tensor respectively.

The state of materials in the nearby zone can be modeled by the Mohr-Coulomb criterion as follows [7, 8].

$$
\sigma_{\theta}=\alpha^{*} \sigma_{r}, \sigma_{r}>>\tau_{0}
$$

where, $\alpha^{*}=\frac{1-\sin \varphi}{1+\sin \varphi}, \tau_{0}$ - cohesion, $\varphi$-mobilized angle of friction.

The displacement change of near-region can be gained by using the mass conservation condition:

$$
\frac{1}{3} \frac{\partial}{\partial r}(r+w)^{3}=\frac{\rho_{0}}{\rho} r^{2} \approx r^{2}
$$

By integrating equation (3) one readily obtains, $\varepsilon_{r}=\frac{\partial w}{\partial r}=\left(\frac{1}{\sqrt[3]{1-\left(\frac{a}{r}\right)^{3}}}\right)^{2}-1, \quad \varepsilon_{\theta}=\sqrt[3]{1-\left(\frac{a}{r}\right)^{3}}-1 \quad$, and from the relation $v=c_{p} \varepsilon_{r}$ at the wave front and the relation $v(a, t)=\dot{a}$ on the surface of the projectiles, it follows that [9]:

$$
v(r, t)=C_{p}^{*}\left(\frac{a}{r}\right)^{2}, v(a, t)=\dot{a}
$$

where, $\mathrm{C}_{\mathrm{p}}$ is the propagation speed of deformational wave which is related to the dimensions of the pulverized granules and the magnitude of volumetric expansion, $\dot{a}$ is the expansion speed of the spherical cavity. 
Substituting equation (2) into equation (1) and integrating equation (1) using relation $\frac{\partial \sigma_{r}}{\partial r}-\mathfrak{R} \frac{\sigma_{r}}{r}=r^{\Re} \frac{\partial}{\partial r}\left(\frac{\sigma_{r}}{r^{\Re}}\right), \mathfrak{R}=-2\left(1-\alpha^{*}\right)$, results in a general solution for the radial stress in the pulverized zone:

$$
\sigma_{r}^{p}=\frac{2}{\mathfrak{R}+4} \rho_{0} v^{2}(r, t)-\frac{2 \dot{a}}{\mathfrak{R}+1} \rho_{0} C_{p}^{*} \frac{a}{r}+r^{\Re} C(t)
$$

where $C(t)$ is an arbitrary function of time, the superscripts $p, f$ and $e$ denote the pulverized, cracking and elastic zones respectively.

The lateral stress, $\sigma_{\vartheta}^{f}=0$, is equal to zero in the cracking zone just like that in a cylinder. Integrating the static equilibrium equation with the condition $-\sigma_{r}^{f}(b)=2 \tau_{s}^{e}$ being considered leads to

$$
-\sigma_{r}^{f}=2 \tau_{s}^{e} \frac{b^{2}}{r^{2}}
$$

Considering the continuity condition of $\sigma_{r}$ across $r=b$, it follows

$$
\sigma_{r}^{p}=\frac{2}{\mathfrak{R}+4} \rho_{0} v^{2}(r, t)\left[1-\left(\frac{r}{b}\right)^{\Re+4}\right]-\frac{2 \dot{a}}{\mathfrak{R}+1} \rho_{0} C_{p}^{*}\left[\frac{a}{r}-\frac{a}{b}\left(\frac{r}{b}\right)^{\Re}\right]
$$

Letting, $\quad \lambda_{s}=2 \tau_{s}^{e}\left(\frac{a}{b}\right)^{\Re}, \quad \lambda_{d}=\frac{2}{\Re+1}\left[1-\left(\frac{a}{b}\right)^{\Re+1}\right], \lambda_{e}=\frac{2}{\Re+4}\left[1-\left(\frac{a}{b}\right)^{\Re+4}\right]$, and considering the condition on the surface of the caliber, equation (7) becomes

$$
\sigma_{n}=-\sigma_{r}^{p}(r=a)=\lambda_{s}+\lambda_{d} \rho_{0} C_{p}^{*} \dot{a}-\lambda_{e} \rho_{0} \dot{a}^{2}
$$

According to equation (3) the deformation is

$$
w^{p, f}(r)=\sqrt[3]{r^{3}-a^{3}}-r
$$

For the elastic zone, from the incompressibility condition $\frac{d w}{d r}+2 \frac{w}{r}=0$, the general expression for the displacement is obtained as $w^{e}=\frac{D}{r^{2}}$, components of strain tensor can be readily calculated as $\varepsilon_{r}^{e}=\frac{\partial w^{e}}{\partial r}=-2 \frac{D}{r^{3}}, \quad \varepsilon_{\theta}^{e}=\frac{w_{e}}{r}=\frac{D}{r^{3}}$, and the equilibrium equation, the stress-strain relation and the boundary condition are as follows

$$
\frac{d \sigma_{r}^{e}}{d r}+2 \frac{\sigma_{r}^{e}-\sigma_{\theta}^{e}}{r}=0, \quad \frac{\sigma_{r}^{e}-\sigma_{\theta}^{e}}{2}=\mu\left(\varepsilon_{r}^{e}-\varepsilon_{\theta}^{e}\right), \quad \sigma_{r}^{e}(R)=0
$$

Solving equation (10) leads to

$$
-\sigma_{r}^{e}=4 \mu D\left(\frac{1}{r^{3}}-\frac{1}{R^{3}}\right)
$$


The parameter $\mathrm{D}$ is determined from the stress condition $\sigma_{r}^{f}(c)=\sigma_{r}^{e}(c)$ at the boundary between the cracking and elastic zones, that is, $D=\frac{\tau_{s}^{e} b^{2} c}{2 \mu\left[1-\left(\frac{c}{R}\right)^{3}\right]}$.

Thus, equation (11) is rewritten as

$$
-\sigma_{r}^{e}=\frac{2 \tau_{s}^{e} b^{2} c}{1-\left(\frac{c}{R}\right)^{3}}\left(\frac{1}{r^{3}}-\frac{1}{R^{3}}\right)
$$

The order for determination of the radii $\mathrm{b}$ and $\mathrm{c}$ of the pulverized and cracking zones, use is made of two conditions as follows. The first condition is displacement continuity at the boundary between the pulverized and elastic zones: $w^{f}(c)=w^{e}(c)$. Considering $w<<c$ at the outer boundary $c$ of the cracking zone, equation (11) is approximated by $w^{f}(c)=a^{3} / 3 c^{2}$. Thus, the aforementioned continuity condition is expressed as

$$
\frac{a^{3}}{3 c^{2}}=\frac{\tau_{s}^{e} b^{2}}{2 c \mu\left[1-\left(\frac{c}{R}\right)^{3}\right]}
$$

The second condition is the energy criterion for the increase of cracks [6], that is, the amount of energy released by the increase of $\mathrm{c}$ is equal to the amount of the effective surface energy $\gamma_{B}$ required by the increase of cracks, whilst the released energy equals the half of the work done by the external forces, i.e. $\frac{1}{2} 4 \pi b^{2}\left|\sigma_{r}^{p}(b)\right| \cdot \Delta w^{p}(b)=2 \gamma_{B}$.n.c. $\Delta c$. From equation (7), equation (9) becomes

$$
\frac{4 \pi b^{2} \tau_{s}^{e}}{n} \cdot \frac{1}{c} \cdot \frac{\partial w^{p}(b)}{\partial c}=2 \gamma_{B}
$$

in which $\gamma_{B}$ is the effective surface energy. Under the plane strain circumstances, $\gamma_{B}=\frac{\pi K_{c}^{2}(1-v)}{4 \mu}$ where $K_{c}$ is the critical stress-intensity factor, $v$ is the poisson's ratio; $n$ is the unit circumference of cracks on the surface of the crack of radius $c, n=6 \pi$ which is identical to the expansion of crack along the three planes across the center of sphere.

Recalling equations (9) and (13) and assuming that $x=\left(\frac{c}{R}\right)^{3}$, $y=\left(\frac{a}{c}\right)^{3}, \beta=6.0 \frac{\tau_{s}^{e}}{G}=6.0 \gamma_{0}, \frac{a}{b}=\frac{1}{2} \sqrt{\frac{\beta}{(1-x) y^{\frac{1}{3}}}}$, the above equation becomes 


$$
-\frac{y^{\frac{5}{3}}(1-x)(1+2 x)}{2 \sqrt{\beta(1-x) y}}\left\{1-\left[1-y(y(1-x) / \beta)^{-\frac{3}{2}}\right]^{-\frac{2}{3}}\right\}=\frac{3 n \gamma_{0} \gamma_{B}}{4 \pi \tau_{s}^{e} a}=\frac{\pi(1-v)}{32} \frac{\beta^{2}}{a / l}
$$

in which, $\gamma_{0}=\frac{\tau_{s}^{e}}{\mu}$ is the limit shear strain, $\Delta=\left(\frac{K_{c}}{\tau_{s}^{e}}\right)^{2}$.

When the derivative of the left hand side of the above equation (negative under stable conditions) equals zone, the critical condition for initiation of instability in the crack zone occurs, that is

$$
\frac{\partial}{\partial c}\left\langle-\frac{y^{\frac{5}{3}}(1-x)(1+2 x)}{2 \sqrt{\beta(1-x) y}}\left\{1-\left[1-y(y(1-x) / \beta)^{-\frac{3}{2}}\right]^{-\frac{2}{3}}\right\}\right\rangle=0
$$

Solving equation (16) leads to $x=0.25$, and substitution of this equation into equation (14) results in relation of $x$ and $c / R$, with which the value of $\eta_{1}$ in the critical state is obtained [10]:

$$
\frac{R_{*}}{a}=\eta_{1} \sqrt{\frac{a}{\Delta}}=\eta_{1} \chi^{2}
$$

The above equation can be regarded as the perforation criterion. From equation (13), it follows:

$$
\frac{a}{b}=\eta_{2} \sqrt[4]{\frac{a}{\Delta}}=\eta_{2} \chi
$$

in which, $\Delta$ represents scaling parameter associated with the characteristic strength parameters, $\chi^{2}=\sqrt{\frac{a}{\Delta}}$.It is the term $\frac{a}{b}$ that represents the transformation relation between different scales for the same media with calibers of different radii.

\section{Formula for calculating penetration depths}

The influence of the term, $\lambda_{e} \rho_{0} \dot{a}^{2}$, in equation (8) on penetration depth is less than $4 \%$ in the case of $v_{0} \leq 1000 \mathrm{~m} / \mathrm{s}$. Therefore, for projectiles with speed of $v_{0} \leq 1000 \mathrm{~m} / \mathrm{s}$, the term, $\lambda_{e} \rho_{0} \dot{a}^{2}$ can be neglected in the computation. The influence of the term, $\lambda_{s}$, in equation (8) on penetration depth is less than $5 \%$ in the case of $v_{0} \geq 400 \mathrm{~m} / \mathrm{s}$. the term, $\lambda_{s}$ can be also neglected in the computation. Equations (8) is thus reduced to

$$
\sigma_{n}=-\sigma_{r}^{p}(r=a)=\lambda_{d} \rho_{0} C_{p} \dot{a}
$$

For any point on the surface of a conical-shaped caliber (the derivation followed can be extended to other shapes), the relation between the radius of the spherical 
cavity, $a$, and the depth $\mathrm{x}$ and the relation between the expansion speed of the spherical cavity $\dot{a}$ and the penetration speed $v$ are as follows

$$
a=x \cdot \operatorname{tg} \theta_{0} / \cos \theta_{0}=x \cdot \frac{\sin \theta_{0}}{\cos \theta_{0}^{2}}, a=v \sin \theta_{0}
$$

The normal force acting over an infinitesimal unit area on the surface of the caliber is $d F_{z}=2 \pi x \sigma_{n} \aleph d x \tan ^{2} \theta_{0}$, letting $\aleph=1+\mu^{\prime} \operatorname{ctg} \theta_{0}$. From equations (8) and (19), one obtains

$$
F_{z}=2 \pi \int_{0}^{l_{d}} x \tan ^{2} \theta_{0} \aleph \sigma_{n} d x=\Sigma \dot{h}
$$

where $\dot{h}$-is speed in terms of penetration depth, positive downwards, $\mu^{\prime}$ - frictional coefficient between the caliber and the rock, $l_{d}=r_{0} \operatorname{ctg} \theta_{0}$ is the length of the caliber, $r_{0}-$ is the radius of the caliber, $\theta_{0}$ - is the semi-angle of the cone, $d$-is the diameter of the caliber, $l_{d} / d$ is the ratio of length to diameter of the caliber. For commonly used calibers,

$$
\begin{aligned}
& \sqrt{1+\left(\frac{l_{d}}{r_{0}}\right)^{2}} / \frac{l_{d}}{r_{0}} \approx 1, \Sigma=d^{2} \frac{1}{\lambda_{1}} \frac{1}{\lambda_{2}} \frac{1}{K_{q}}, \lambda_{1}=\frac{\sqrt{1+\operatorname{ctg}^{2} \theta_{0}}}{\pi \aleph} \approx \frac{\operatorname{ctg} \theta_{0}}{\aleph \pi}, K_{q}=\frac{1}{\rho_{0} C_{p}^{*}}, \\
& \lambda_{2}=2(\Re+1) /\left[1-\frac{8}{\mathfrak{R}+9}\left(\eta_{2} \chi\right)^{\Re+1}\right]=\left(2 \alpha^{*}-1\right) /\left[1-\frac{8}{2 \alpha^{*}+7}\left(\eta_{2} \chi\right)^{\left(2 \alpha^{*}-1\right)}\right] .
\end{aligned}
$$

The differential equation of motion of the projectile is

$$
m \ddot{h}=-F_{z}=-\Sigma \dot{h}
$$

From the above equation together with the initial conditions $h(t=0)=0, \dot{h}(t=0)=v_{0}$, the penetration depth $h$ is derived as

$$
h=\frac{m}{d^{2}} \lambda_{1} \lambda_{2} K_{p} v_{0}
$$

\section{Conclusions}

(1) The analytical model proposed in this paper pinpoints reasonably and straightforwardly the inherent behavior of deformation and failure of rock under penetration of projectiles.

(2) The penetration depths of projectiles into rock computed by the proposed method have a wide range of applications.

\section{References}

[1] Heuze, F. E., “An Overview of Projectile Penetration into Geological Materials, with Emphasis on Rock", in "Computational Techniques for Contact, Impact, Penetration and Perforation of Solids", eds. Schwer, L. E. et al., AMD-Vol.103, ASME, pp.275-308, 1989. 
[2] Kennedy, R. P., A Review of Procedures for the Analysis and Design of Rock Structures to Resist Missile Impact Effect. Nucl. Eng. Des., 3 (37), pp.183-203, 1976.

[3] Canfield, J. A. and Clator, I. G., "Development of a Scaling Law and Techniques to Investigate Penetration in Concrete", NWL Report No. 2057, U.S. Naval Weapons Laboratory, Dahlgren, VA ,1966.

[4] Forrestal, M. J., Altman, B. S., Cargile, J. D. and Hanchak, S. J., “An Empirical Equation for Penetration Depth of Ogive-Nose Projectiles into Concrete Targets". Int. J. Impact Eng., 15(4), pp.395-405, 1994.

[5] Forrestal, M. J. et al., "Penetration of Grout and Concrete Targets with Ogive-Nose Steel Projectiles”. Int. J. Impact Eng., 18(5), pp.465-476, 1996.

[6] Mastilovic, S. and Krajcinovic, D., "Penetration of Rigid Projectiles Through Quasi-brittle Materials". ASME J. Appl. Mech., 15(66), 585$592,1999$.

[7] N. Morozov, Y. Petrov, Dynamics of fracture, Springer-Verlag: Berlin and New York, pp.110-120, 2000.

[8] A .Carpinteri A.R. Ingraffea, YANG Yu-hui, WANG Yu-zhen, Fracture mechanics of concrete Material characterization and testing, the Press of Hunan university: Hunan, pp.168-169, 1988.

[9] Wang Mingyang, Deng Hongjian, Qan Qihu, Study on the Problems of Near the Cavity of Explosion and Penetration in Rock. Chinese Journal of Rock Mechanics and Engineering, 24(16), pp.2859-2863, 2005.

[10] Wang Mingyang, Zheng Daliang, Qian Qihu, The scaling law problems of penetration and perforation for projectile into concrete media. Explosion and Shock Waves, 24(1), pp.7-15, 2004. 